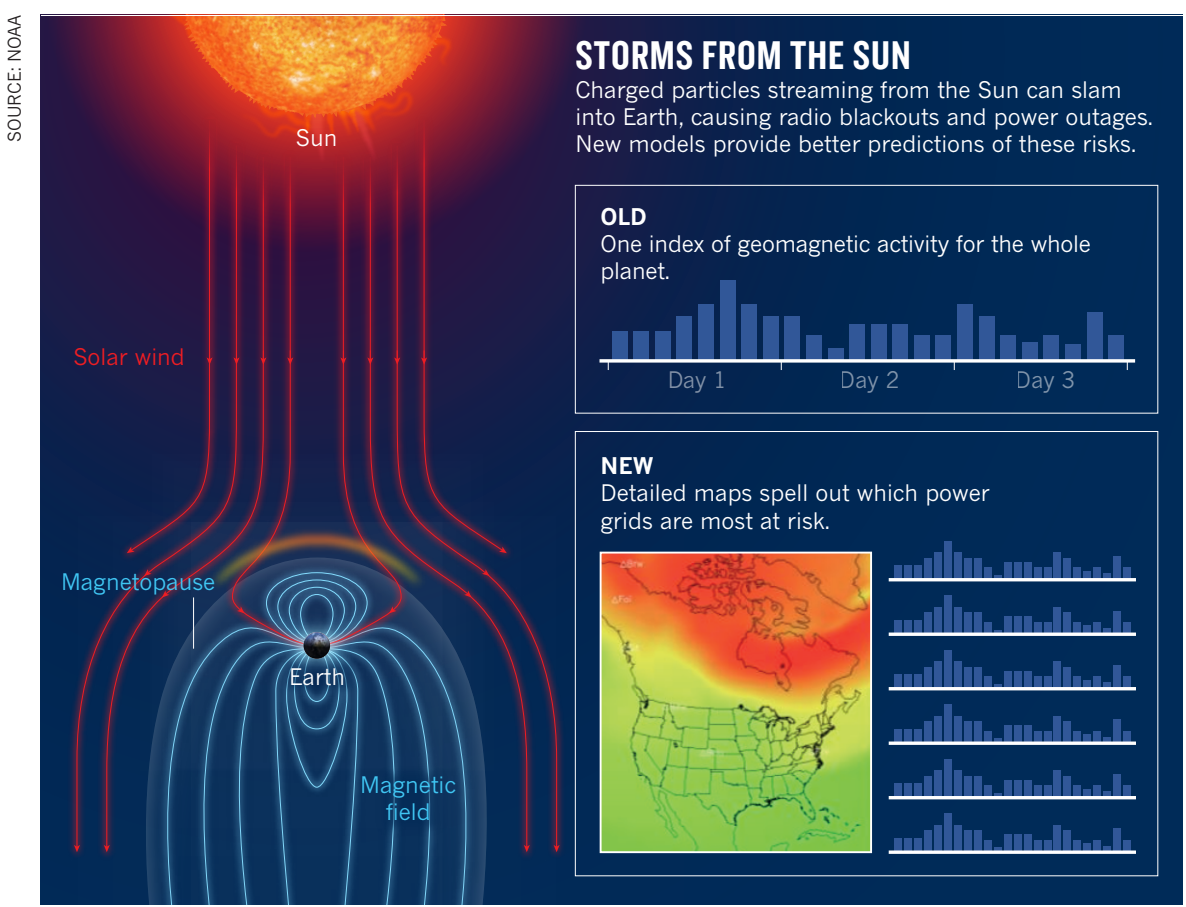

that ranks the current geomagnetic threat to the entire Earth. The new 'geospace' forecast, which draws on more than two decades of research, comes in the form of a map showing which areas are likely to be hit hardest (G. Tóth et al. J. Geophys. Res. Space Phys. 110, A12226; 2005).

Knowing that Canada, for instance, will be hit harder than northern Europe helps grid operators, says Tamas Gombosi, a space physicist at the University of Michigan in Ann Arbor who helped to develop the model. $\mathrm{He}$ compares it to having a hurricane forecast that says a storm will hit Florida, rather than just somewhere on the planet (see 'Storms from the Sun').

\section{MAGNETOSPHERE MODEL}

Space-weather forecasting is as rudimentary as conventional weather forecasting was three or four decades ago, says Catherine Burnett, space-weather programme manager at the UK Met Office in Exeter. Researchers have developed different models to describe various portions of the Sun-Earth system, but linking them into a coherent framework has been difficult. The Michigan approach combines 15 models that collectively describe the solar atmosphere through interplanetary space and into Earth's magnetic realm. The NOAA forecast incorporates three of those: one model describing Earth's entire magnetosphere, another focusing on the inner magnetosphere and one for electrical activity in the upper atmosphere.

The inner magnetosphere chunk is crucial to the model's overall success, says developer Gábor Tóth at the University of Michigan. It describes how energetic particles flow and interact as they approach Earth's poles, and how the particles affect magnetism at the planet's surface. Alerts can provide roughly 20 minutes to one hour of warning.

NOAA's improved forecasts are part of a push by US agencies to implement a national space-weather strategy issued last year by the White House. Regulators will also soon require power-grid operators to produce hazard assessments that include the threat of solar storms. "Without those two pieces, we wouldn't have remotely the interest we have now," says Antti Pulkkinen, a space-weather researcher at NASA's Goddard Space Flight Center in Greenbelt, Maryland. "It really has changed the game."

NOAA plans to continue refining its forecasts as new research rolls in. The possible improvements include incorporating how the geology beneath power grids affects the intensity of a solar storm. Fluctuating magnetic fields can induce electrical currents to flow in the ground, which sets up further problems for transmission lines. "All of this is terrifically complicated," says Jeffrey Love, a geomagnetics researcher at the US Geological Survey in Golden, Colorado.

In their latest paper, Love, Pulkkinen and their colleagues describe the most detailed map of these 'geoelectric hazards' across part of the United States (J. J. Love et al. Geophys. Res. Lett. http://doi.org/bqpm; 2016). Of the areas surveyed so far, those at the highest risk are the upper Midwestern states of Minnesota and Wisconsin, where complex geology induces strong electrical currents.

Adding in 3D models of these ground currents will improve the next generation of NOAA forecasts, Rutledge says. "This is by no means the end."

\section{ASTRONOM}

\section{Billion-star map unveiled}

\section{Gaia mission reveals Milky Way is bigger than thought.}

\section{BY DAVIDE CASTELVECCHI}

$\mathrm{T}$ he European Space Agency (ESA) has released the largest, most detailed map yet of the Milky Way. It pinpoints the 3D positions of 1.1 billion stars, almost 400 million of which were previously unknown to science. ESA's Gaia space observatory mapped out the preliminary catalogue, which is based on its first 14 months of data-taking. The results are expected to transform astronomy - allowing researchers to discover new extrasolar planets, examine the distribution of dark matter, fine-tune models of how stars evolve and refine their measurements of the Universe's rate of expansion (see Nature 537, 292-293; 2016).

Hundreds of astronomers began to access the database as soon as it was publicly released on 14 September, says Gaia project scientist Timo Prusti, who works at ESA's European Space Research and Technology Centre in Noordwijk, the Netherlands. Within the first 24 hours, 11,000 users had downloaded data, ESA adds. The mission, which launched in 2013 and cost nearly $€ 1$ billion (US $\$ 1$.1 billion), has already found more stars than researchers expected, which suggests that the Milky Way is slightly bigger than previously estimated, says Gisella Clementini, a Gaia researcher at the Bologna Astronomical Observatory in Italy. But few new results were announced at the catalogue's unveiling; Gaia's team was allowed to do only limited analyses before the data release.

One notable result is a measurement of the distance of the Pleiades, a cluster of stars in the constellation Taurus that is the subject of a long-running controversy (see Nature http://doi.org/bqpt; 2014). Numerous measurements put the Pleiades cluster at a distance of about 135 parsecs (440 light years) from the Sun, but Gaia's predecessor, ESA's Hipparcos mission, found it to be about 15 parsecs closer. Gaia measured 134 parsecs, give or take 6 parsecs - suggesting that the Hipparcos findings were inaccurate. With more years of observation, Gaia's measurements will be able to pinpoint the distances of many of the Galaxy's stars to within $1 \%$. "What Gaia is going to do is going to be phenomenal," says Wendy Freedman, an astronomer at the University of Chicago in Illinois. "It will be the fundamental go-to place for astronomers for decades to come."

See go.nature.com $/ 2$ cevbnq for a longer version of this story. 